\title{
Twisted multilayer graphene exhibiting strong absorption bands induced by van Hove Singularities
}

\author{
UMESHA MOGERA ${ }^{\mathbf{1}}$ and GIRIDHAR U KULKARNI ${ }^{1,2, *}$ \\ ${ }^{1}$ Centre for Nano and Soft Matter Sciences, Jalahalli P.O., Bangalore 560013, India \\ ${ }^{2}$ On lien from Jawaharlal Nehru Centre for Advanced Scientific Research, Jakkur P.O., Bangalore 560064, India \\ *Author for correspondence (guk@ cens.res.in)
}

MS received 26 February 2018; accepted 3 May 2018; published online 21 September 2018

\begin{abstract}
Twisted bilayer graphene exhibits several angle-dependent properties due to the emergence of the van Hove Singularities in its density of states. Among them, twist-angle-dependent optical absorption has gained a lot of attention due to its presence in the visible spectral region. However, observation of such absorption is experimentally tricky due to large transmittance. In this study, we use highly decoupled twisted multilayer graphene to observe such absorption in the visible region using a simple spectrometer. A large number of twisted graphene layers in the system enable observation of such absorption evident in the visible region; the absorption band position correlates with the twist angle measured using selective area electron diffraction pattern as well as predictions from theory. While the Raman spectra were akin to those of the decoupled graphene system, at specific twist angle of $\sim 13^{\circ}$, the spectrum contained clear signatures of G-band enhancement.
\end{abstract}

Keywords. Graphene; twisted bilayer graphene; twisted multilayer graphene; optical absorption; G-band enhancement; van Hove Singularities.

\section{Introduction}

The twisted bilayer graphene (tBLG), two graphene layers mis-oriented with a twist angle $\theta\left(0^{\circ}<\theta<30^{\circ}\right)$, has attracted a lot of interest in recent years due to its unique angledependent properties [1-4]. The interlayer coupling between two graphene layers in $\mathrm{tBLG}$ can be tuned with the twist angle and at higher angles $\left(\theta>10^{\circ}\right)$, the layers become effectively decoupled from one another [5]. The twist angle, $\theta$, adds a new degree of freedom to the system, generating several $\theta$-dependent electronic and optical properties that are nonexistent in single-layer graphene (SLG) [6-8]. For example, a twisted system possesses a logarithmic divergence in its density of states, giving rise to van Hove Singularities (vHSs) [9-11] and a strong Raman G band enhancement at specific angles $[12,13]$.

Another important property of the tBLG is that they exhibit absorption bands in the otherwise featureless absorption of SLG due to the appearance of vHSs. The positions of absorption maxima are directly related to the energy difference between vHSs and hence the twist angle [14]. Though well studied theoretically, it has always been challenging to observe such absorption bands, perhaps due to weak intensity of such bands that merges with huge transmittance of bilayer graphene ( $\sim 94 \%)$. Recently, using a specially designed UV-vis-NIR hyperspectral microscope, Havener et al [15] extracted angle-dependent absorption from complex optical conductivity. Using the optical colour contrast method, Robinson et al [3] have shown the appearance of visible colour in the tBLG. However, direct observation of twist-induced absorption using a simple spectrometer has not been easy due to limited size of twisted graphene as well as limited absorption of tBLG. What is desirable is to have a wide stack of decoupled layers.

Recently, we have pioneered the synthesis of a new variant of graphene species, namely, twisted multilayer graphene (tMLG) grown by modified CVD technique $[16,17]$. The unprecedented characteristic of the tMLG is its high degree of decoupling arising from interlayer twists [18]. In spite of hundreds of layers, its Raman spectrum exhibits a single Lorentzian narrow 2D band (width $\sim 16-24 \mathrm{~cm}^{-1}$ ) and high $I_{2 \mathrm{D}} / I_{\mathrm{G}}$ values (up to 16 ), importantly with complete absence of ' $D$ ' peak. In short, tMLG represents many sets of tBLG with many rotated graphene layers in it. Due to the large path length possible, tMLG offers a platform to study emergence of absorption features measurable using a simple spectrometer. 


\section{Experimental}

Detailed synthesis procedure of tMLG has been reported elsewhere [16]. Briefly, a polycrystalline $\mathrm{Ni}$ foil $(7 \mu \mathrm{m}$, 99.9\%, Advent Research Materials) was cut into a $4.0 \times 0.6 \mathrm{~cm}^{2}$ strip and connected to the current carrying electrodes. Naphthalene solution $(10 \mu 1,1 \mathrm{mM})$ in chloroform was drop cast onto the $\mathrm{Ni}$ foil and allowed to dry. After reaching a rotary vacuum of $\sim 4$ mTorr, the $\mathrm{Ni}$ foil was Joule heated to red hot $\left(\sim 900^{\circ} \mathrm{C}\right)$ with a current density of $120-150 \mathrm{~A} \mathrm{~mm}^{-2}$ using a DC source for $15 \mathrm{~min}$ and then immediately cooled. The graphene grown on $\mathrm{Ni}$ was transferred onto a glass substrate by etching underlying Ni using $1 \mathrm{Maq}$. $\mathrm{FeCl}_{3}$ solution, followed by scooping away floating graphene flake using the glass substrate.

The absorption spectra were recorded in the transmission mode using a Perkin Elmer Lambda 900 UV-vis-NIR spectrometer. To precisely record absorption spectra from a region, a 1-mm-diameter pinhole was placed on the sample. Raman spectra were acquired in backscattering geometry using the $532 \mathrm{~nm}$ excitation wavelength (Horiba XploRA ONE Raman microscope) with the laser power of $\sim 2-5 \mathrm{~mW}$ at the sample surface. To measure the thickness of the tMLG, an optical profiler (OP) (Wyko NT9100, Veeco, USA) was used.
For transmission electron microscopy (TEM), graphene on $\mathrm{Ni}$ was transferred onto a holey carbon film of the $\mathrm{Cu}$ grid by chemically etching of $\mathrm{Ni}$ using $1 \mathrm{M} \mathrm{FeCl}_{3}$ solution. Selected area electron diffraction (SAED) was performed using a Technai F30 UHR operating at $200 \mathrm{kV}$. High-resolution TEM images were obtained using a FEI Talos. FFT patterns were generated from TEM images using Digital Micrograph software, Gatan Inc.

\section{Results and discussion}

The synthesis of tMLG involves Joule heating of metal catalyst in the presence of an aromatic solid hydrocarbon source followed by rapid cooling (rate: $50-100^{\circ} \mathrm{C} \mathrm{s}^{-1}$ ) (see figure 1a). Unlike conventional top-down approach of stacking graphene layers requiring sophisticated instrumentation, the present bottom-up approach offers a rapid and facile way to produce such twisted graphene over an area of $>2 \mathrm{~cm}^{2}$ (see figure $1 \mathrm{~b}$ ). Further, these graphene stacks can be transferred onto a substrate by simple etching procedures. In figure 1c, one such transferred graphene stack (size of $\sim 10 \mathrm{~mm}^{2}$ ) is shown. A remarkable property of the stack is the Raman spectrum resembling that of a high-quality SLG in spite (a)

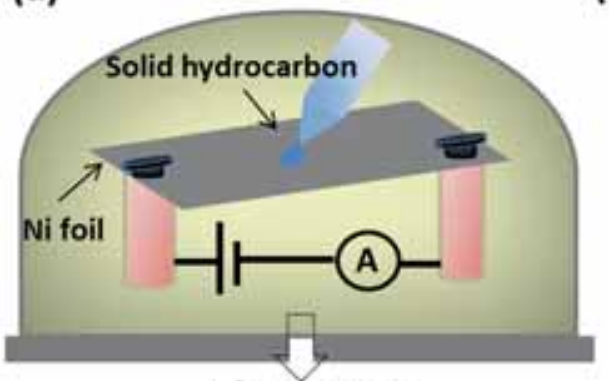

rotary vacuum (b)

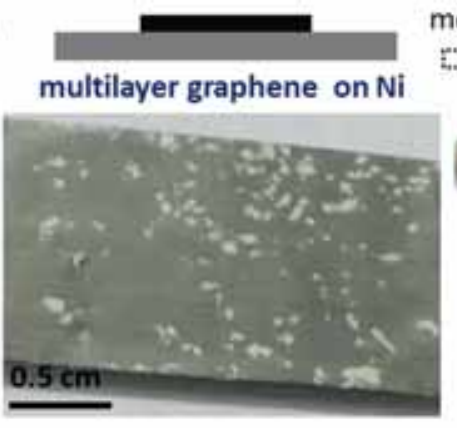

(e)

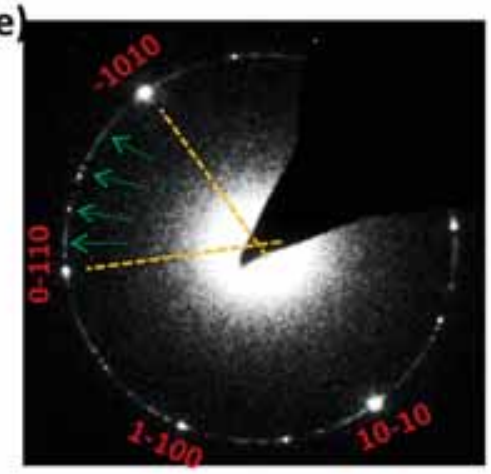

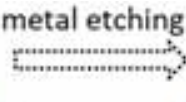

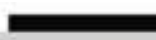

(c)

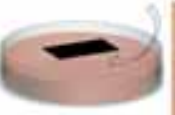

multilayer graphene on glass

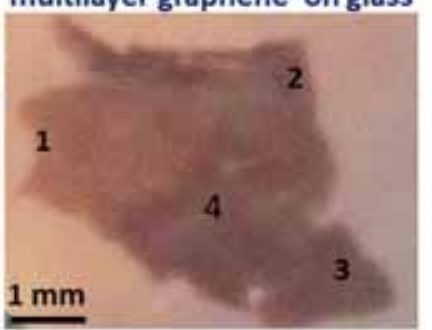

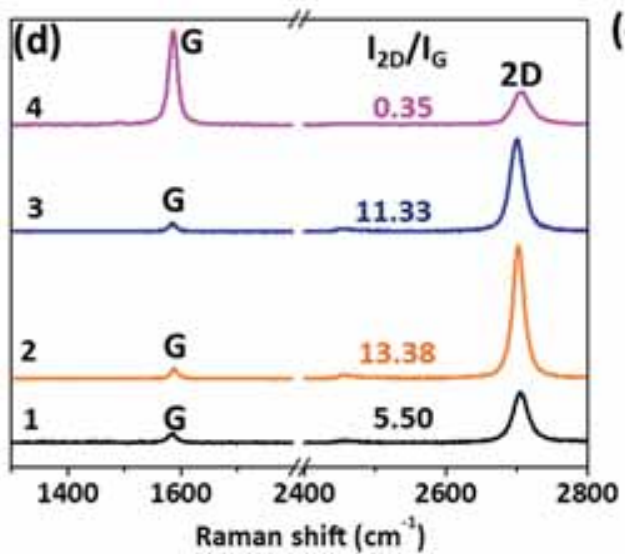

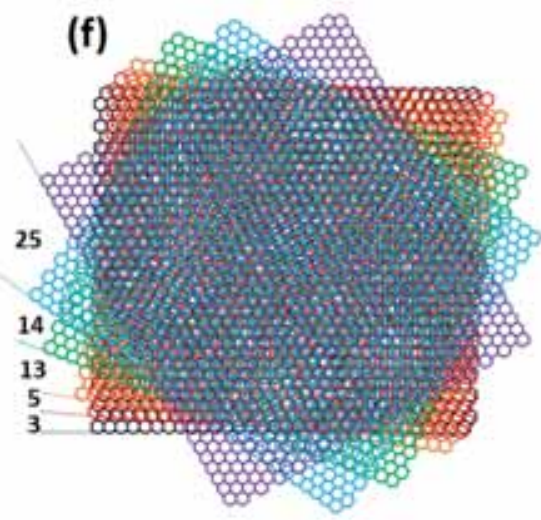

Figure 1. Synthesis and properties of tMLG. (a) Schematic of tMLG synthesis procedure involving Joule heating of a metal catalyst in the presence of aromatic solid hydrocarbon sources. A photograph of (b) tMLG grown on Ni foil and (c) tMLG transferred onto a glass substrate. (b) Representative Raman spectra acquired on the regions indicated by numbers in c. The $I_{2 \mathrm{D}} / I_{\mathrm{G}}$ ratios are indicated. (e) SAED pattern of a tMLG showing the presence of diffraction spots (arrows) in between the hexagonal spots (dashed line). (f) Schematic of random twisted graphene layers in tMLG. 
of the thickness of tens of nanometres (see figures $1 \mathrm{~d}$ and supplementary figure $\mathrm{S} 1$ ). In all the spectra, the ' $\mathrm{D}$ ' band is completely absent and the $2 \mathrm{D}$ band is essentially a single symmetric Lorentzian with FWHM in the range of $16-24 \mathrm{~cm}^{-1}$. The $I_{2 \mathrm{D}} / I_{\mathrm{G}}$ value was found to be in the range 3-16, much higher than reported for supported SLG. In our previous study [16], we have stated that such behaviour is due to decreased interlayer interactions among the layers to an extent that the layers behave as though they are freely suspended. The unusual angular relation among adjacent layers, similar to tBLG case, is the primary reason for diminished interlayer interactions. The selective area electron diffraction (SAED) pattern from a tMLG sample transferred onto TEM grid (figure 1e) shows intermittent diffraction spots (see arrows), which could be directly related to angular rotations among the layers as shown schematically in figure 1f. While many twist angles are found, it is unlikely that adjacent layers are rotated by zero twist (AB stacking).
Given the accessible area and thickness, we attempted to measure optical absorption spectra for tMLG using a simple UV-vis spectrometer. The absorption spectrum in figure $2 \mathrm{a}$ from a tMLG exhibits an absorption band at $\sim 540 \mathrm{~nm}$, strikingly different from the featureless spectrum from typical SLG [19,20] (see the red curve in figure 2a). To the best of our knowledge, this is the first experimental observation of a visible band from twisted graphene system, measured directly by passing a light beam. Previous attempts to experimentally capture such absorption features of tBLG required specially designed imaging techniques, perhaps due to weak intensity of the bands overlapping with the high transmittance $(\sim 94 \%)$ of the bilayer graphene. In the present tMLG, such absorption was observable in the UV-vis spectrometer due to large sampling of twisted graphene layers $\left(1 \mathrm{~mm}^{2}\right.$ area and $12-15 \mathrm{~nm}$ thickness). This absorption band appears due to twist-angle-induced vHSs as shown schematically in figure $2 b$. An important aspect of the vHSs is that their energy
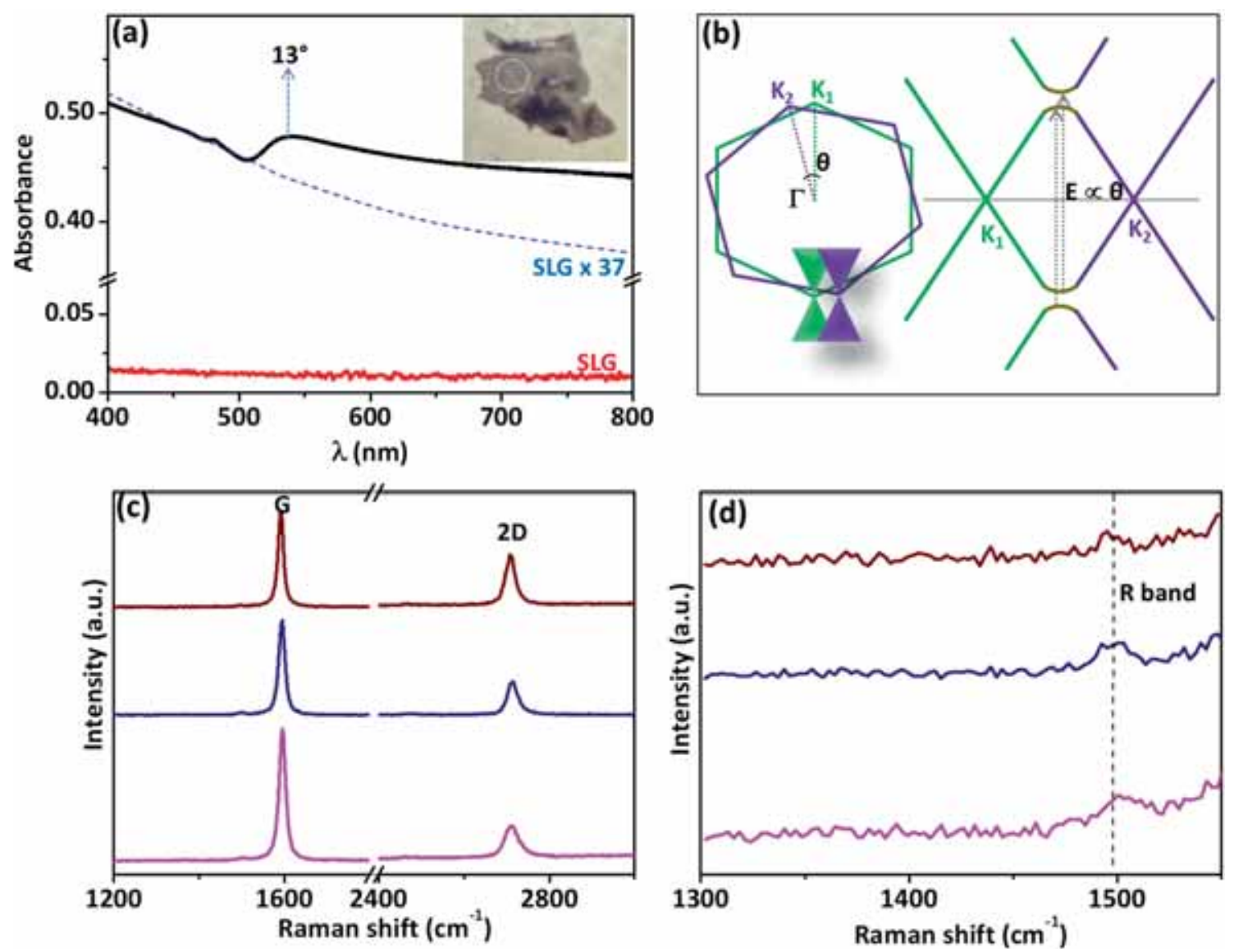

Figure 2. Absorption spectrum of tMLG. (a) The absorption spectrum of tMLG measured over visible range $(400-800 \mathrm{~nm})$. The arrow points to the absorption maximum with correlated twist angle. Inset is the photograph of the tMLG flake. The dotted circle indicates the region on which absorption spectrum was acquired. Red curve is the absorption spectrum acquired in SLG. Blue dashed line is the absorption spectrum obtained by adding SLG 37 times. The number 37 was chosen to match the absorption at lower wavelengths. This could also indicate that the region under study contained nearly 37 layers. (b) A schematic representing the twist-induced vHSs in tBLG. (c) Raman spectra acquired over three regions of tMLG $\left(\lambda_{\mathrm{ex}}=532 \mathrm{~nm}\right)$. (d) Magnified portion of the Raman spectra over the region of $1300-1550 \mathrm{~cm}^{-1}$. Dotted bar indicates the position of $\mathrm{R}$ band at $\sim 1500 \mathrm{~cm}^{-1}$. 
difference varies monotonically with the twist angle [14]. By correlating the position of absorption maxima with the twist angle, the predominant twist angle in present tMLG may be assigned to $\sim 13^{\circ}$. However, other rotations are also possible. This is clearly evident in the broad absorption beyond $500 \mathrm{~nm}$ on comparing with the background absorption (blue dashed line), the latter calculated as a multiple of SLG absorption (see figure $2 \mathrm{a}$ ). This broad absorption may be assigned to rotations of lower angles, while a peak at $\sim 480 \mathrm{~nm}$ may be related to rotation of $16^{\circ}$. Since many twist angles are included in the tMLG, various energy positions of vHSs get summed up and thus, no single peak may be expected to appear. However, a predominant rotation (for example, $13^{\circ}$ in the present sample) is evident. However, at higher energies, the absorption spectrum follows that of SLG as SLG itself has its own absorption at higher energies.

It is also known that the tBLG exhibits G-band enhancement due to rise of the vHSs in tBLG [12,13]. Interestingly, the G-band enhancement varies with twist angle and laser excitation energy $\left(\lambda_{\text {ex }}\right)$. For example, when using $\lambda_{\text {ex }}$ of $532 \mathrm{~nm}, \mathrm{G}$ band is enhanced when $\theta=13^{\circ}$. In the present study, this observation was used for confirming the twist angle. Three representative Raman spectra, measured on the same tMLG flake, are shown in figure $2 \mathrm{c}$, where the $2 \mathrm{D}$ band intensity is noticeably diminished but continued to be a single symmetric Lorentzian, implying that this observation in not due to any graphitic nature. The relative enhancement of $\mathrm{G}$ band (2-5 times) with respect to that of 2D is clearly due to another known process, the G-band resonance or enhancement $[12,13]$. In support of this, the appearance of a new band, called $\mathrm{R}$ band, around $\sim 1500 \mathrm{~cm}^{-1}$, was examined as the position of this band is known to vary with twist angle [14]. The magnified region in $1300-1550 \mathrm{~cm}^{-1}$ clearly shows a small intensity in the region of the $\mathrm{R}$ band (figure $2 \mathrm{~d}$ ). Its position $\left(\sim 1500 \mathrm{~cm}^{-1}\right)$ matches the value expected for a twist angle of $13^{\circ}[14]$.

Further, the twist angle between graphene layers was confirmed by TEM analysis (see figure 3 ). Figure $3 \mathrm{a}$ is the low-magnification TEM image of a tMLG flake. A portion of it is magnified in figure $3 \mathrm{~b}$, which shows parallel strip-like features as marked by the dotted orange lines. Along with the parallel lines, one can also see hexagonal structures in the marked region. Figure 3c shows the arrangement of carbon atoms along the black coloured line. Such arrangement of carbon atoms was observed throughout the TEM image (see supplementary figure S2), which implies a high degree of 2D crystallinity of the tMLG. From TEM image, a FFT (Fast Fourier Transform) pattern was generated as shown in figure $3 \mathrm{~d}$. The main diffraction spots corresponding to the hexagonal lattice have split into sharp spots with angular spread of $2-3^{\circ}$. In addition to these spots, many intermediate spots are also seen but with milder intensity. Interestingly, when two lines are drawn meeting the main and intermediate diffraction spots
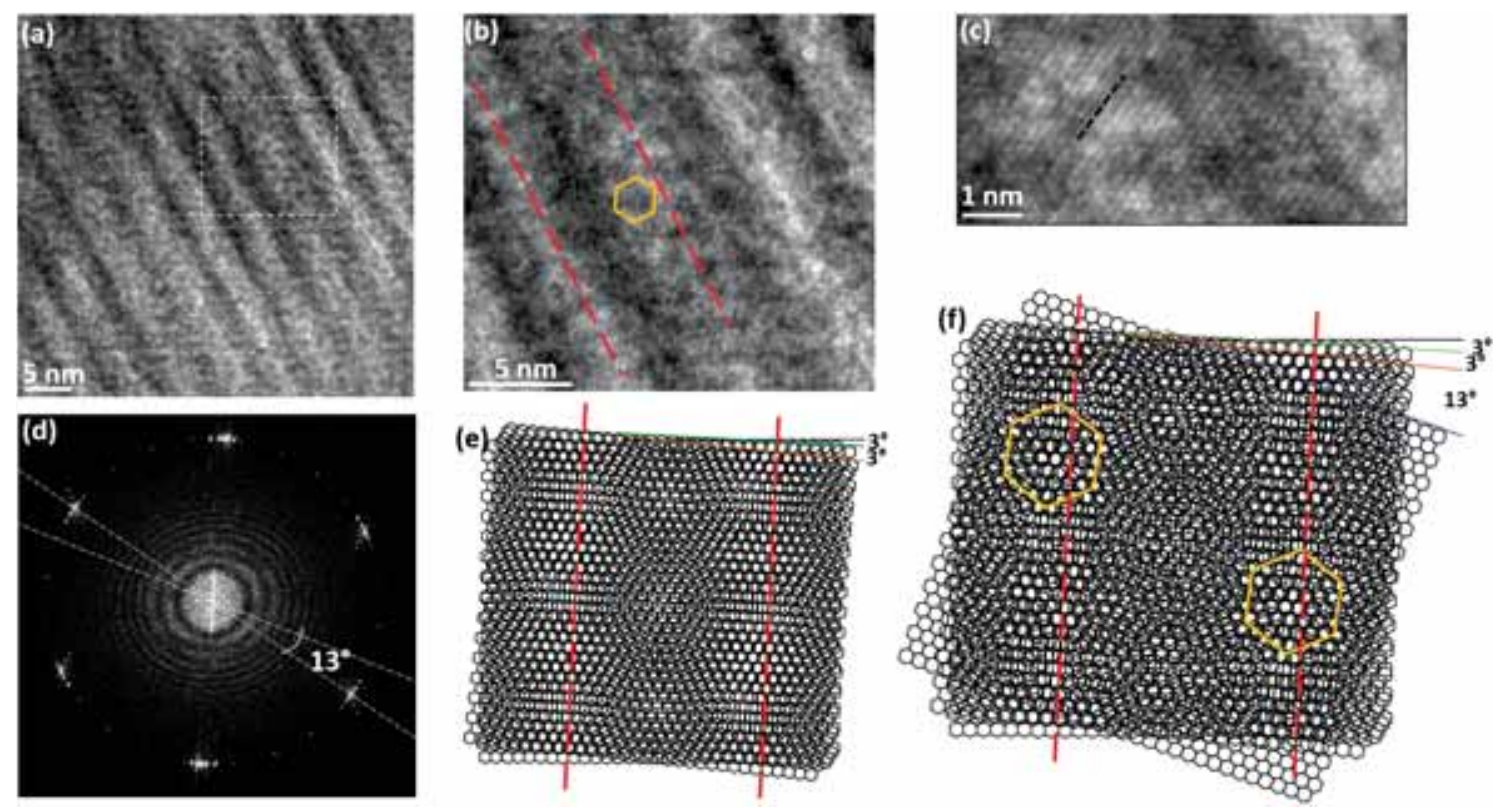

Figure 3. TEM analysis of tMLG. (a) TEM image of tMLG flake. (b) High-resolution TEM of magnified portion indicated by the dotted square box in a. (c) A portion of the image is magnified to see the lattice arrangement of atoms along the drawn dashed line. (d) FFT image generated from $\mathbf{a}$. The lines are drawn at an angle of $13^{\circ}$ meeting diffraction points. (e, f) Schematic representation of rotations of graphene layers. Here, first three layers shown in e are rotated at an angle of $3^{\circ}$ with respect to adjacent layers and the fourth layer in $\mathbf{e}$ is rotated at an angle of $13^{\circ}$ with respect to the 3rd layer. The orange lines are shown resembling the appearance of strips in $\mathbf{a}, \mathbf{b}$ and golden colour hexagons are drawn resembling the hexagonal pattern in $\mathbf{b}$. 

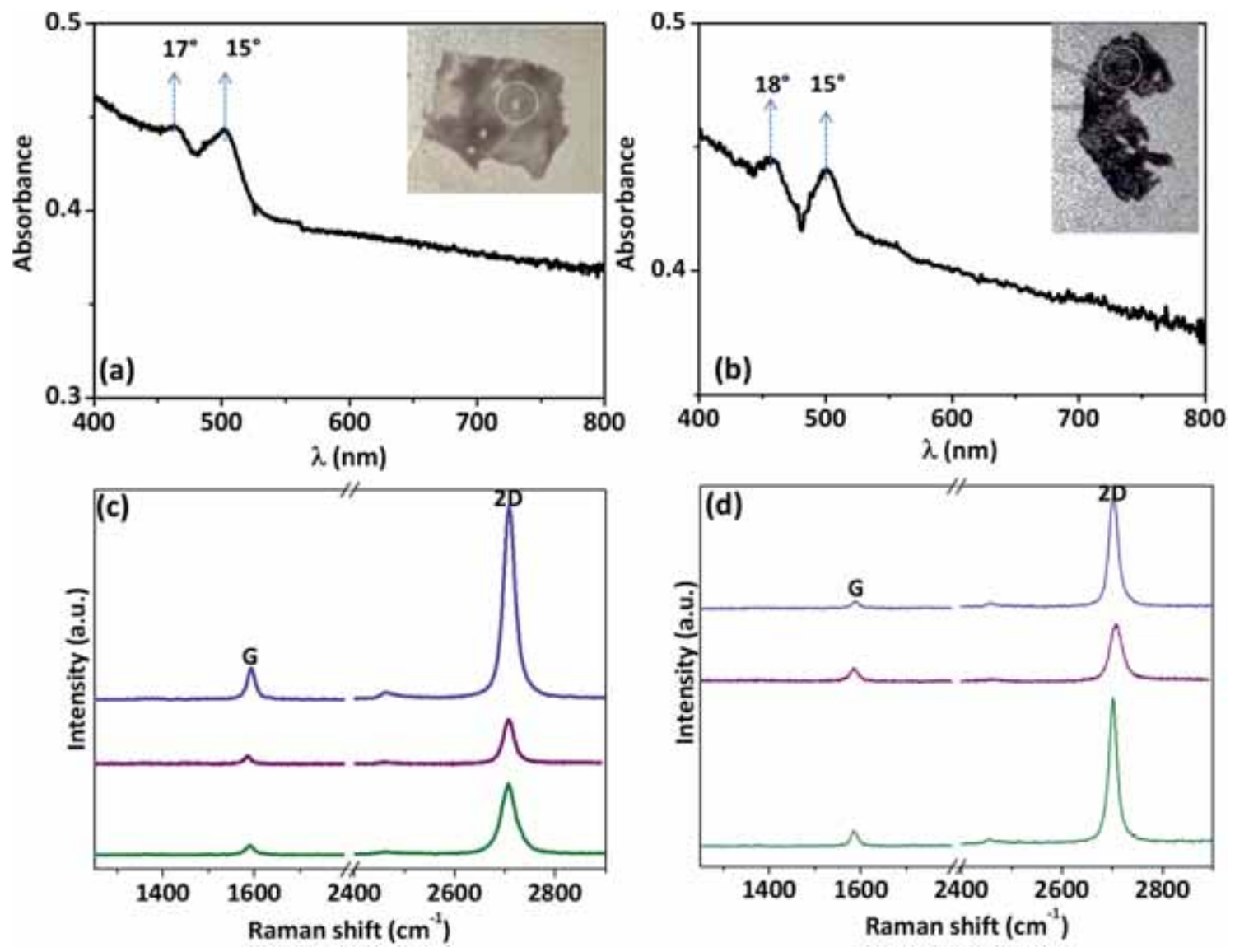

Figure 4. The absorption band observed in other samples. The absorption spectra measured from sample (a) tMLG 2 and (b) tMLG 3. Insets are photograph of the samples. Dotted circles represent the region on which spectrum has been acquired. The Raman spectra acquired on (c) tMLG 2 and (d) tMLG 3.

to measure the angle between them, it turns out to be $\sim 13^{\circ}$.

Following the FFT analysis, two schematic models were drawn. In the first model (see figure 3e) three graphene layers are rotated with respect to adjacent layers at an angle of $3^{\circ}$, which generates parallel strip-like features with a periodicity of $\sim 5 \mathrm{~nm}$. The observed periodicity nearly matches the periodicity of strip-like structures seen in the TEM micrograph (see figure $3 b$ ). In the second model (see figure 3f), another layer was added to a rotation of $13^{\circ}$ with respect to the third layer. This resulted in hexagonal patterns with width $\sim 1.6 \mathrm{~nm}$, similar to the one observed in TEM in figure $3 \mathrm{~b}$ (indicated by yellow coloured hexagons). These analyses further confirm the presence of graphene layers with the $13^{\circ}$ twist in the tMLG sample.

Such observation of absorption bands was not limited to one sample (see figure 4). Another sample (tMLG 2) exhibited dual absorption maxima at 460 and $500 \mathrm{~nm}$ corresponding to the twist angles of $17^{\circ}$ and $15^{\circ}$, respectively (figure $4 \mathrm{a}$ ). Another sample (tMLG 3) showed similar features (455 and $505 \mathrm{~nm}$ ) with twist angles of $18^{\circ}$ and $15^{\circ}$ (figure $4 \mathrm{~b}$ ). Accordingly, the Raman spectra showed signatures of single-layer characteristics with $I_{2 \mathrm{D}} / I_{\mathrm{G}}$ values higher than with single
Lorentzian 2D peak (see figure $4 \mathrm{c}$ and $\mathrm{d}$ ). The presence of such high $I_{2 \mathrm{D}} / I_{\mathrm{G}}$ values confirms the twist angle of $>15^{\circ}$ seen in the absorption spectra. It may be noted that the absorption maxima appear at different positions in three different samples depending on predominant rotations present. In the tMLG, since there is little control over twist angles among layers (the same situation in CVD-grown twisted graphene), different samples tend to show different absorption features depending on dominant twist angles present in that particular sample. Also, multiple absorption spectra could be observed from a given sample when acquired at different locations as the twist angles at different locations may vary due to polycrystalline nature of graphene layers in tMLG.

\section{Conclusion}

In the present study, using tMLG grown by modified CVD technique, the absorption features were detected using a simple spectrometer. Since tMLG represents many sets of tBLG in it, the absorption bands become prominently observable in conventional UV-vis measurements. In one sample (tMLG 1), correlating the position of absorption bands with the 
theoretical model [14], the twist angle was assigned to $\sim 13^{\circ}$. The TEM image and electron diffraction pattern of tMLG 1 clearly show the presence of graphene layers with the $13^{\circ}$ twist in the sample. The G-band enhancement and the presence of $\mathrm{R}$ band corroborate the degree of twist in the sample. Importantly, absorption features corresponding to varied twist angles were routinely observed in all measured samples.

\section{Acknowledgements}

We thank Prof C N R Rao for his constant encouragement and support. This research work is supported by Department of Science and Technology (DST), New Delhi, India. UM acknowledges IGSTC project for the fellowship.

\section{References}

[1] Yan W, Meng L, Liu M, Qiao J B, Chu Z D, Dou R F et al 2014 Phys. Rev. B 90115402

[2] Landgraf W, Shallcross S, Türschmann K, Weckbecker D and Pankratov O 2013 Phys. Rev. B 87075433

[3] Robinson J T, Schmucker S W, Diaconescu C B, Long J P, Culbertson J C, Ohta T et al 2013 ACS Nano 7637

[4] Jorio A and Cançado L G 2013 Solid State Commun. 1753

[5] Luican A, Li G, Reina A, Kong J, Nair R R, Novoselov K S et al 2011 Phys. Rev. Lett. 106126802
[6] Ding Y, Peng Q, Gan L, Wu R, Ou X, Zhang Q et al 2016 Chem. Mater. 281034

[7] Xian L, Wang Z F and Chou M Y 2013 Nano Lett. 13 5159

[8] Wang Z F, Liu F and Chou M Y 2012 Nano Lett. 123833

[9] Li G, Luican A, Lopes dos Santos J M B, Castro Neto A H, Reina A, Kong J et al 2010 Nat. Phys. 6109

[10] Liao L, Wang H, Peng H, Yin J, Koh A L, Chen Y et al 2015 Nano Lett. 155585

[11] Yin J B, Wang H, Peng H, Tan Z J, Liao L, Lin L et al 2016 Nat. Commun. 710699

[12] Havener R W, Zhuang H, Brown L, Hennig R G and Park J 2012 Nano Lett. 123162

[13] Ni Z, Liu L, Wang Y, Zheng Z, Li L J, Yu T et al 2009 Phys. Rev. B 80125404

[14] Carozo V, Almeida C M, Fragneaud B, Bedê P M, Moutinho M V O, Ribeiro-Soares J et al 2013 Phys. Rev. B 88 085401

[15] Havener R W, Liang Y, Brown L, Yang L and Park J 2014 Nano Lett. 143353

[16] Mogera U, Dhanya R, Pujar R, Narayana C and Kulkarni G U 2015 J. Phys. Chem. Lett. 64437

[17] Mogera U, Kurra N, Radhakrishnan D, Narayana C and Kulkarni G U 2014 Carbon 78384

[18] Mogera U, Walia S, Bannur B, Gedda M and Kulkarni G U 2017 J. Phys. Chem. C 12113938

[19] Bae S, Kim H, Lee Y, Xu X, Park J S, Zheng Y et al 2010 Nat. Nanotechnol. 5574

[20] Hobeom K, Sang-Hoon B, Tae-Hee H, Kyung-Geun L, Jong-Hyun A and Tae-Woo L 2014 Nanotechnology 25 014012 